\title{
Design of a Portable Health Monitoring System Based on Node MCU
}

\author{
R.K.Parate, S.J.Sharma
}

\begin{abstract}
In the present work, we have designed a health monitoring system based on Node MCU to monitor temperature, heart rate and oxygen saturation level $\left(\mathrm{SpO}_{2}\right)$ signals, sensed by respective sensors. The necessary signal conditioning circuits have been designed in our laboratory using off-the shelf electronic components. A Data acquisition system has been designed using ESP 32 Node MCU. The designed system is a low-cost alternative to the commercially available USB controller based health monitoring systems. Firmware has been developed and deployed into the Node MCU using arduino IDE. The acquired data has been displayed on OLED display. The result shows maximum errors in the measured parameters within $2 \%$. The designed system helps to achieve portability, high functionality and low cost which makes it an easy accessible tool for public, hospital, sports healthcare and other medical purposes.
\end{abstract}

Keywords: Temperature, heart rate and $\mathrm{SpO}_{2}$, Data acquisition system, Node MCU, USB controller, OLED display

\section{INTRODUCTION}

Innovations in Electronics have deeply percolated in our everyday life, and the medical field is not a special case. With growing public concern and government demands for reforms [1], in the online monitoring devices, the need for well equipped hospitals and diagnostic centers are increasing continuously due to more awareness of people about their health. More than 1.8 million patients in India pass on due to heart illness out of which $42.7 \%$ die while transit to diagnostic center due to absence of appropriate checking in the vehicle carrying the patient. Hence, the improvement of Health monitoring systems plays major role in modern day life [2]. Health care monitoring systems which monitor the patient's health continuously and transmit the vital data to the doctors or hospitals are in great demand. Such monitoring and diagnostic devices are mainly used in hospitals for the examination of patients [3]. Heart rate, Oxygen saturation level $\left(\mathrm{SpO}_{2}\right)$ and temperature are the three basic parameters which are routinely monitored by the

Revised Manuscript Received on December 30, 2019.

* Correspondence Author

R.K.Parate*, Department of Electronics, S.K.Porwal College, Kamptee, Rashtrasant Tukadoji Maharaj Nagpur University Nagpur,India. Email: rkparate@yahoo.co.in

Dr. S.J.Sharma, Department of Electronics and Computer Science, Rashtrasant Tukadoji Maharaj Nagpur University, Nagpur, India. Email: rkparate@yahoo.co.in

(c) The Authors. Published by Blue Eyes Intelligence Engineering and Sciences Publication (BEIESP). This is an open access article under the CC BY-NC-ND license (http://creativecommons.org/licenses/by-nc-nd/4.0/) medical experts to get the state of human health. Heart rate was in the past estimated by setting the thumb over the blood vessel throb, and counting the pulses usually in a 30 second period. Heart rate was then found by multiplying the obtained number by 2 . This method although simple, is not an exact and can give blunders when the pulse rate is high [4]. ECG is one of the as often as possible utilized and exact methods for measuring the heart rate. But ECG is not an affordable method for poor people [5]. The heart rate of a healthy adult at rest is around 72 beats per minute (bpm). Athletes ordinarily have more heart rates than less dynamic individuals. Infants have a much higher heart rate at around $120 \mathrm{bpm}$, while older have heart rates at around $90 \mathrm{bpm}$. Heart rate varies significantly between individuals based on wellness, age and sexual orientation [6]. On the other hand, the percentage of arterial blood saturated with oxygen helps to decide the viability of a patient's respiratory system. In prior days, $\mathrm{SpO}_{2}$ was estimated by taking samples of blood and measuring $\mathrm{O}_{2}$ levels legitimately. This method was invasive and was not able to give online estimation. Because of this, $\mathrm{SpO}_{2}$ was not perceived as a significant measure of wellness until a non-invasive method of measuring it in real-time (pulse oximetry) was established [7]. Based on measuring the absorption of red and infrared light that passes through a patient's finger or ear lobe using light sensors. The normal acceptable ranges of $\mathrm{SpO}_{2}$ are from 95 to 100 percent. The allowable range of $\mathrm{SpO}_{2}$ for patient having hypoxic drive problem lies between 88 to 92 percent [3]., [8]. Due to its non-invasive nature, high accuracy, and sensible cost, optical pulse oximeter are widely adopted as a standard patient monitoring techniques. Temperature is a measure of the degree of heat intensity. Human body temperature is an expression of its molecular excitation. Body temperature is maintained through a balance of the heat produced by the body and the heat lost from the body. Body temperature likewise differs from individual to individual for the duration of the day. The body temperature is seen as lowest in the early morning while it is highest during the early night, so It is important to seen the progressions routinely [9]. Peoples are homoeothermic and body temperature is regulated at about $37^{\circ} \mathrm{C} \pm 1^{\circ} \mathrm{C}$. The fundamental purpose behind looking at body temperature is to hunt for any indication of foundational disease or inflammation in the presence of a fever. Temperature depression needs to be evaluated. It is also important to review the pattern of variations of the patient's temperature. A patient with a fever of $38{ }^{\circ} \mathrm{C}$ does not really give threatening indication, if his past temperature has been higher. Monitoring human body temperature comes under preliminary clinical examination [10]. 


\section{Design of a Portable Health Monitoring System Based on Node MCU}

In the present work, we have designed portable, low cost device that monitor various biomedical signals like heart rate, $\mathrm{SpO}_{2}$ and body temperature.

\section{EXPERIMENTAL}

Figure 1 shows the functional block diagram of the proposed Health monitoring system. The system is built around Node MCU ESP 32 along with temperature sensor DS18B20, Pulse oximetry sensor MAX 30100 and 0.96"' OLED display.

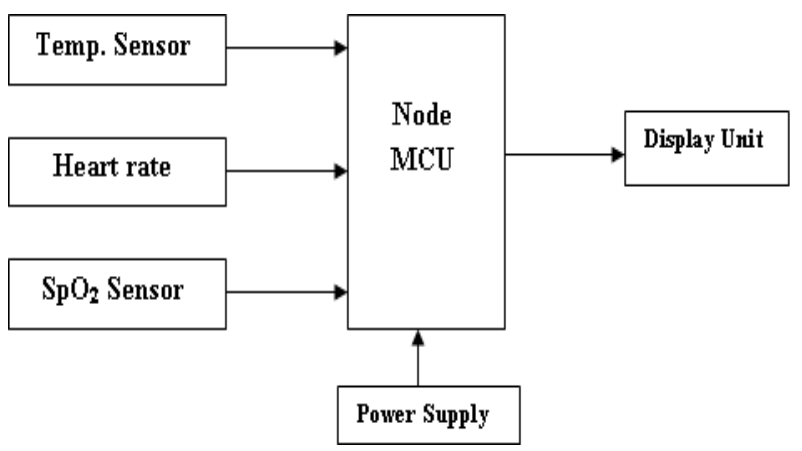

Fig. 1: Block Diagram of the System

\subsection{Node MCU (ESP32) Microcontroller (ESP32)}

ESP 32 is low cost, low power system on chip microcontroller with integrated $\mathrm{Wi}-\mathrm{Fi}$ and dual mode Bluetooth. Controller (ESP32) is created and intended for wearable electronics, cell phones and internet of things (IOT) capacities. It provides the features of low power consumption. ESP32 controller is awaken from time to time when a specific condition is recognized. Low duty cycle is used to reduce the utilization of energy that the chip dissipates. It is basically designed to satisfy the better power and RF generation, performance robustness, power utilization, reliability in a huge basis of acquisition, power scenes and flexibility.

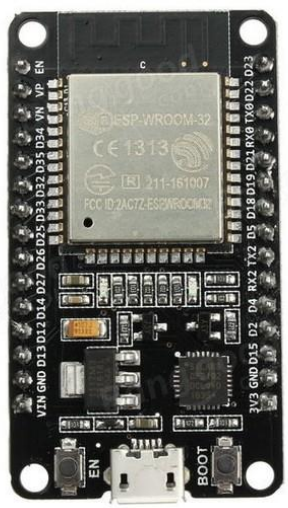

Fig.2 ESP 32 Node MCU

\subsection{Temperature Sensor (DS18B20)}

DS18B20 digital temperature sensor gives 9 bit to 12 bit Celsius temperature estimations and gives indication with fixed user programmable higher and lower trigger points. Sensor interface with single wire bus that according to definition needs just a single data line for correspondence with a main processor. Likewise, this sensor can get power directly from the data line ("parasite control"), which eliminates the requirement of external power supply. Each
DS18B20 has a one of a kind 64 bit sequential code, which enables numerous DS18B20s to work on a similar 1-Wire bus. In this way, it is easy to use one microcontroller to control various DS18B20s distributed over an enormous zone. Applications that can profit by this element incorporate environmental controls, temperature monitoring systems inside equipments, process monitoring and control systems.

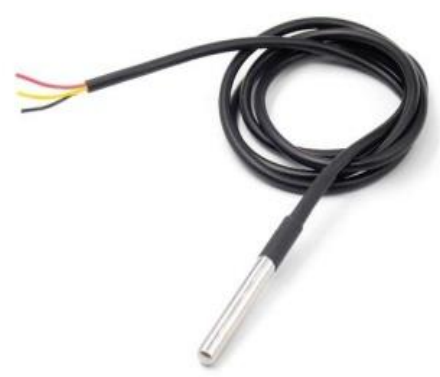

Fig.3 Temperature sensor

\subsection{Pulse oximetry and Heart rate sensor}

MAX30100 is incorporated with pulse oximetry and heart rate sensor framework arrangement intended for the requesting prerequisites of wearable gadgets. It consolidates two LEDs, a photodetector, optimized optics, and low noise anolog signal processing to recognize $\mathrm{SpO}_{2}$ and heart rate. MAX30100 works from $1.8 \mathrm{~V}$ and $3.3 \mathrm{~V}$ power supply and can be powered down through programming with insignificant standby current, allowing the power supply to stay associated consistently. MAX30100 provides exceptionally little absolute arrangement size without sacrificing optical or electrical performance. Minimum external hardware components are required to design portable device. MAX30100 is completely configurable through programming registers, and the digital data is stored in a 16 deep- FIFO register inside the sensor. The first in first out register enables sensor to be associated with a controller on a shared bus, where the information isn't being read continuously from the device registers.

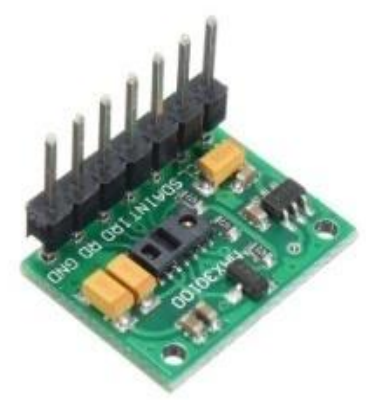

Fig.4 Pulse oximetry and Heart rate sensor

\subsection{OLED:}

OLED (Organic Light-Emitting Diode) is a self light-emitting innovation made out of a thin, multi-layered organic film placed between an anode and cathode.

Published By:

Blue Eyes Intelligence Engineering 
In comparison with LCD innovation, OLED does not need a back illumination which provides better contrast in dark environment.

This altogether decreases the power required to run the OLED and is the reason the presentation has such high differentiation, very wide viewing angle and can show profound dark levels. OLED has high application potential for basically wide range of displays and is viewed as definitive innovation for up and coming age of flat-panel displays. OLED has four pins and speaks with any microcontroller utilizing I2C correspondence convention.. Due to I2C communication it provide simple connection between microcontroller and OLED.

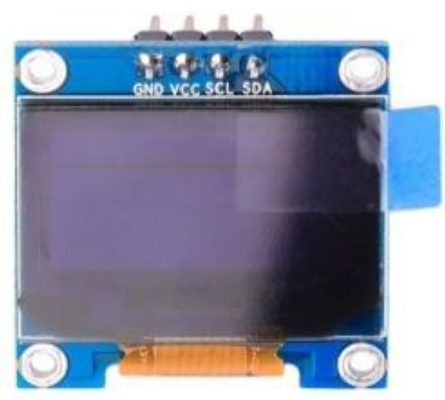

Fig.5 OLED

\subsection{Power Supply}

There are three parts that need the power supply association. One is simply the Node MCU and other two are the sensor modules communicating with the Node MCU. It's quite straightforward in build simply required two distinctive yield voltages that is 5 volt and 3.3 volt, or else we can simply give 5.0 volt to the Node MCU and give 3.3 volt to the sensors from the Node MCU breakout board.

\section{PROPOSED SYSTEM}

System has been constructed using above hardware components. Sensors cannot be directly interfaced with Node MCU, Hence there is need to connect some passive components externally for use with Node MCU. DS 18B20 temperature sensor is prepared in parasitic mode by connecting $4.7 \mathrm{~K} \Omega$ resistance between power source and data line which eliminates the need for external power supply. Similarly MAX 30100 prepared for use with Node MCU by removing three $4.7 \mathrm{~K} \Omega$ resistances of breakout boards. The three $4.7 \mathrm{~K} \Omega$ resistances are connected externally between SCL, SDA, INT with respect to 3.3V. Arduino is an open source platform used for developing this electronic system. Appropriate libraries for both the sensors and OLED has been downloaded from online sources. Code is developed in Arduino platform and uploaded into the Node MCU. Now sensors have been connected to the subjects and data displayed on OLED display. A complete designed prototype is shown in fig. 6 .

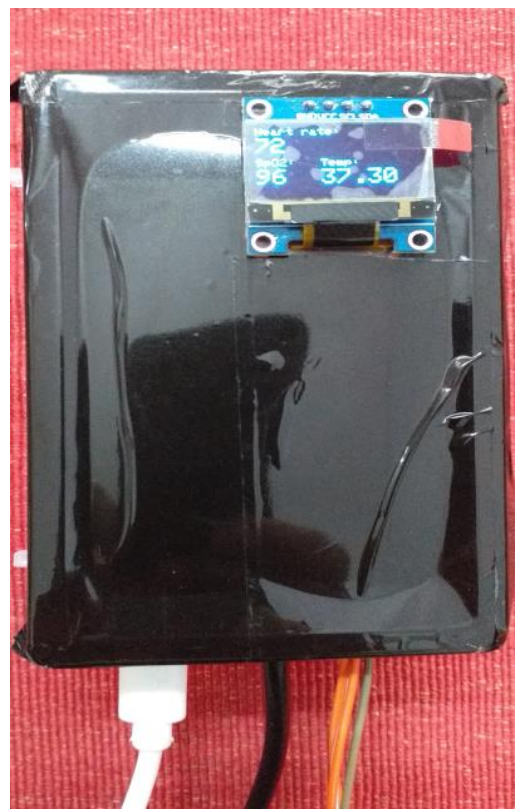

Fig.6 System Prototype

\section{RESULTS AND DISCUSSION}

The designed system has been tested in the hospital for clinical trials on 25 subjects of different age groups. Comparison between commercially available and designed system for monitoring Heart rate, $\mathrm{SpO}_{2}$ and Temperature has been done. The performance of system is satisfactory. The system is compact, portable and user friendly. This system is applicable for family, hospital centers, community medical treatment, sports healthcare and other medical purposes.

\section{CONCLUSION}

A Node MCU based portable Health Monitoring system is designed to measure Heart rate, $\mathrm{SpO}_{2}$ and temperature. The system is cheaper due to home monitoring of patients at home as compared to the expensive workplaces. Results from designed system are quite impressive as compared to those standard commercially available systems. However accuracy could be improved by using high quality sensor IC with much better $\mathrm{S} / \mathrm{N}$ ratio and better peak detection algorithm. The system can also be designed to alert a person for indication of some abnormalities. The person who has no technical knowledge can also operate this device.

\section{REFERENCES}

1. R. Sunitha and K. Prathyusha, "Microcontroller based Heartbeat, blood pressure and body temperature monitoring and alerting system using GSM modem”, IOSR J. Elect. \& Comm. Engg., vol. 9, no.6, pp. 100-104, (2014).

2. P. Bharahan, V. Nadar and S. Wayal, " Remote Health Monitoring system using IOT”, Int. J. Adv. Res. Ideas \& Innov. in Tech. vol..3, no.2, pp. 23-24, (2017).

3. S. Sali and C. Parvathi, "Integrated Wireless Health Monitoring System for Elderly People” Int. J. Innov. Res. Com. \& Commu. Engg. vol. 5, no. 4, pp. 480-490, (2017).

4. E. Jahan, T. Barrua and U. salma, "An Overview on Heart rate monitoring and pulse oximeter system”, Int. J. lat. Res. Sci. \& Tech. vol. 3, no. 5, pp .148-152, (2014). 
5. E. Dogo, F. Sado and S. Adah , "Design of a Simple and Low-Cost Microcontroller Based Medicare Device for Heart Beat Monitoring", African J. Comp. \& ICT, vol.6, no.5, pp. 121-128, (2013).

6. S. Das, "The Development of a Microcontroller Based Low Cost Heart Rate Counter for Health Care Systems" Int. J. Engg. Trends \& Tech. vol.4, no.2, pp. 207-211, (2013).

7. K. Ajith , B. George, B. Aravind and K .Martin, "Integration of low cost Spo2 sensor in wearable monitor", ARPN J. Engg \& appl. Sci. vol.10, no.17, pp. 7553-7558, ( 2015).

8. D. Kaur, S. Kumar and S. Sharma, "Online Graphical Display of Blood Oxygen Saturation and Pulse Rate", Int. J. Sci. \& Engg. Res. vol. 2, no.6, pp. 1-5, (2011).

9. B. Oyebola, O. Oluremi, and T. Odueso, “ Development of a Heartbeat and Temperature Measuring System for Remote Health Nursing for the Aged in Developing Country" Sci. J. Cir. Sys. Sig. Pro.vol.7, no.1, pp. 34-42, (2018).

10. F. Sudhindra ,S. Annarao, R. Vani and P. Hunagund, " Development of Real Time Human Body Temperature (Hypothermia \& Hyperthermia) Monitoring \& Alert System with GSM \& GPS”, Int. J. Innov. Res. Sci. Engg. \& Tech.vol. 5, no.6, pp. 9355-9362, (2016).

11. R. Shariff and H. N. Suresh, "Wearable Vital Signs Monitoring System", Int. J. Engg. \& Adv. Tech. Vol.6, no.5, pp. 116-119, (2017).

12. A. Prabhakar, S. Oza and C. Gautam, "IOT and Wearable Devices", Int. J. Engg. \& Adv. Tech. Vol.8, no.5, pp. 1705-1707, (2019).

13. R. Priyanka and M. Reji, "IOT based Health Monitoring system using Blynk App”, Int. J. Engg. \& Adv. Tech. Vol.8, no.6, pp. 78-81,(2019).

14. Maxim integrated, MAX 30100 pulse oximeter and heart rate sensor ICs data sheet www.datasheetspdf.com/max30100.

15. www.datasheetspdf.com/ds18b20.

16. www.RandomNerdTutorials.com

\section{AUTHORS PROFILE}

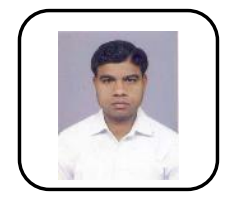

R. K. Parate currently working as Assistant professor in S. K. Porwal College Kamptee 440001 and Research Scholar in Deapartment of Electronics and Computer Science, Rashtrasant Tukadoji Maharaj Nagpur University, Nagpur. He is pursuing doctoral work in Electronics under the faculty of Science \& Technology.

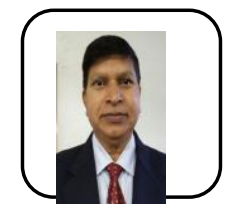

Dr. S. J. Sharma, Professor and Head, Department of Electronics and Computer Science, Rashtrasant Tukadoji Maharaj Nagpur University, Nagpur, 440033. He has 31 Years teaching experience and 27 Years Research Experience. 83 research paper have been published in peer reviewed journals and 132 papers have been presented at various conferences. 12 students have been awarded Ph.D. degree under his guidance. His area of interest is virtual instrumentation, ultrasonic and embedded system design. 